\title{
Análisis espacial basado en SIG del malestar percibido ante industrias contaminantes: el caso del complejo industrial ventanas, Chile
}

\author{
Antonio Moreno-Jiménez y \\ Manuel Fuenzalida-Díaz
}

\section{Resumen}

Los efectos ambientales derivados de actividades humanas ocasionan los llamados campos de externalidades en el entorno de ellas, manifestándose bien como emisiones o impactos físicos, bien como vivencias negativas afectando a la población. La medición de ese malestar social y su expresión espacial constituye el asunto central de esta contribución, en la que se aborda el caso de los residentes próximos a un gran complejo industrial en Chile. A tal fin se diseñó y realizó una encuesta domiciliaria para determinar la percepción de varios impactos ambientales, que luego fue georreferenciada. Para establecer la magnitud del malestar socio-espacial se recurrió a la técnica de los estimadores de densidad núcleo (kernel), que permiten generar con SIG superficies y expresivos mapas representativos de las diversas experiencias negativas, así como un indicador sintético de las mismas. Con ello se pretende aportar una vía para aprehender, en cantidad y en lugar, esos elusivos efectos y concienciar de su trascendencia.

Palabras clave: Malestar socio-espacial. Percepción ambiental. Contaminación . Externalidades. Densidad focal kernel

\section{Abstract}

The environmental effects of human activities shape the so-called externality fields around them, portrayed either as emissions and physical impacts, or as negative experiences affecting population. The measurement of this social bad-being and its spatial expression is the main issue of this contribution, where the case of residents near a large industrial complex in Chile is tackled. To this end a home survey, lately geo-referenced, was designed and achieved to determine the perception of various environmental impacts. To elucidate the amount of socio-spatial bad-being a kernel density estimator was applied, provided it enables to obtain GIS based surfaces and expressive maps representing the various negative experiences, and a synthetic 
index of them too. In this way it is intended to provide a way to capture, both in quantity and place, these elusive effects and to spread awareness about it.

Keywords: Socio-spatial bad-being. Environmental perception. Pollution. Externalities, kernel density.

Antonio Moreno-Jiménez (antonio.moreno@uam.es), Departamento de Geografía, Universidad Autónoma de Madrid, España. Manuel Fuenzalida-Díaz (mfuenzal@uahurtado. cl), Departamento de Geografía, Universidad Alberto Hurtado, Chile.

Trabajo realizado parcialmente en el marco del proyecto CSO2011-26177 "Contaminación atmosférica urbana y justicia ambiental: metodología de evaluación y estudio de casos con sistemas de información geográfica", financiado por el Ministerio de Ciencia e Innovación de España.

Trabajo realizado parcialmente en el marco del proyecto FONDECYT No 11121354 , financiado por la Comisión Nacional de Investigación Científica y Tecnológica (CONICYT) de Chile. 


\section{INTRODUCCIÓN}

Los problemas ambientales que afectan a la sociedad constituyen una preocupación prioritaria, por cuanto con frecuencia ocasionan efectos de gran trascendencia social, económica, política, etc. Uno de los frentes más sugerentes del mismo concierne a la aprehensión de los efectos o impactos que fenómenos o procesos (sean naturales o antrópicos) ocasionan sobre la población. Su medición y análisis constituye una necesidad preferente, puesto que permite plantear políticas, planes y medidas de gestión. La multiplicidad de los tipos de efectos derivados (a menudo asimilables a externalidades espaciales) ha requerido adoptar instrumentos de obtención y tratamiento de datos muy diversos. Así junto a indicadores de tipo objetivo (e.g. basados en medidas instrumentales o estadísticas), el recurso a otros de naturaleza subjetiva (i.e. basados en la percepción o sensación humana) resulta habitual.

La evaluación de aspectos ambientales a partir de las percepciones humanas conforma una vía apropiada para aproximarse al grado de bienestar o malestar sentido y a su geografía. En ese sentido los estímulos provenientes del entorno pueden ser captados, identificados y valorados por los ciudadanos, posibilitando así la determinación de los conocidos "campos de externalidades" que emergen alrededor de los focos emisores, sea cual sea su naturaleza (Pinch, 1985; Moreno y Escolano, 1992: 214-218). Ello abre la puerta a una cartografía y análisis espacial de esas apreciaciones sociales sobre hechos ambientales y, por extensión, a una determinación de perjuicios o costes, a veces soslayados u ocultados. Cuando se trata de externalidades generadas por actividades humanas, por ejemplo las productivas, su evaluación desde la subjetividad personal y su localización espacial es la condición necesaria para poder dar respuestas fundadas sobre los componentes del malestar ocasionado (costes externos), y la forma como se reparten social y espacialmente. El abordaje de la percepción de la contaminación atmosférica, como describen Catalán-Vázquez y Jarillo-Soto (2010), ha sido realizado desde planteamientos paradigmáticos muy diferentes (positivistas, teoría crítica, constructivistas), que resultan complementarios en sus logros.

El presente trabajo, incardinado en esta línea de indagación, ya de notable recorrido, propone un procedimiento para la medición y plasmación espacial de los efectos ambientales (externalidades) percibidos por los residentes próximos a un gran complejo industrial y la aplica a un estudio de caso de Chile, adoptando una aproximación o paradigma calificable de geo tecnológico (vid. Buzai, 2011; Moreno, 2010 y 2013). En el siguiente apartado se presenta el marco conceptual del análisis y los objetivos, para luego exponer las fuentes de información y los métodos 
adoptados, en los que el recurso a técnicas de análisis espacial y a la tecnología SIG proporciona la base metodológica fundamental. A continuación se describen algunos de los hallazgos alcanzados y se termina con una discusión de los mismos y conclusiones.

\section{CONTEXTO TEÓRICO Y ANTECEDENTES}

Es tradicional en las investigaciones geográficas considerar que la mente humana genera imágenes subjetivas del medio natural, que terminan teniendo gran importancia en el comportamiento espacial de los individuos (v.gr. Gould y White, 1974). Estas imágenes son producto de la sensación inmediata y del recuerdo de experiencias anteriores, que las personas utilizan para interpretar la información proveniente del medio exterior y orientar la acción. Según Lynch (1960), esta imagen mental no sólo es una forma de comprender el espacio para lograr una orientación del mismo, sino que también se asocia a sentimientos y emociones vinculadas al espacio vivido, de allí la importancia que tiene cada lugar para el sujeto.

La Geografía cuenta con herramientas para tratar esta cuestión: el mapa mental, que es una representación subjetiva del ambiente. Buzai (2011) clasifica los mapas mentales de acuerdo a varios autores que han incursionado en esta aproximación: elementos visuales (K. Lynch), preferencias (P. Gould) y comparaciones (C. de Castro). De ellos, es de interés aquí mencionar el de preferencias, en donde Gould (1975) establece seis líneas diferentes de abordaje para el estudio de la percepción y la valoración ambiental: (1) percepción de recursos, (2) percepción de paisajes, (3) percepción del riesgo, (4) percepción del espacio, (5) percepción del ambiente urbano y (6) percepción como evaluación ambiental. En este último se estudia de qué manera la población tiende a aprehender y evaluar diferentes sectores del espacio geográfico, cuyo tratamiento con técnicas utilizadas por la geografía cuantitativa (i.e. cuadrados / cuadrantes, densidad núcleo o kernel, interpolación), permite analizar superficies de percepción que se convierten en importantes herramientas para la toma de decisiones.

En esa línea, se le atribuye utilidad al mapa mental principalmente en tres aspectos (Catling, 1978):

- conforma un instrumento de diagnóstico, ya que es una manifestación del nivel de desarrollo espacial;

- es una fuente de información, puesto que permite conocer los diversos elementos significativos en las imágenes mentales, y

- se considera un producto de instrucción, debido a que orienta y da indicios para la creación cartográfica. 
Ahora bien, el término percepción ambiental encierra diferentes acepciones dependiendo de la disciplina dominante que consulte el investigador al intentar caracterizar el comportamiento humano. Psicología, Sociología y Geografía han generado un nicho de trabajo multidisciplinar. Para nuestra disciplina el centro de atención está en la relación hombre-medio y las interacciones espaciales que se producen, intentando desvelar el proceso específico por el cual ciertos fenómenos se registran claramente, mientras otros, se pierden en las sombras o se eliminan (Tuan, 1974).

Barroto et al. (2011) distinguen cuatro facetas relevantes en la percepción ambiental:

1. Visión del medio ambiente y de los problemas ambientales: Ideas del individuo sobre el medio ambiente y sus dimensiones, además de los problemas ambientales y su extensión.

2. Responsabilidad ambiental: Apreciación de los individuos sobre los agentes causantes de problemas ambientales y aquellos agentes que deben intervenir para evitarlos.

3. Actitud ambiental y decisión de incorporarse al cambio: Predisposición del pensamiento humano a actuar en favor o en contra del entorno social, teniendo en consideración sus vivencias, experiencias, conocimientos y valores con respecto a su entorno.

4. Formación medioambiental: Impresión que tienen los individuos sobre la calidad y efectividad de las acciones de educación ambiental.

La percepción ambiental se puede considerar como la base para las decisiones de manejo y conservación del medio natural; al mismo tiempo puede contribuir al diseño de políticas públicas orientadas a redirigir los procesos de deterioro ambiental.

De cara a la evaluación ambiental en términos de percepción de peligros o emisiones de actividades o focos (e. g. contaminantes), resulta imprescindible incorporar el concepto de bienestar. Siguiendo a Moreno (1998), la geografía del bienestar resulta, a menudo, inseparable de la del malestar. La atención al tema atraviesa las fronteras disciplinares y así en la ciencia política, economía regional, geografía, derecho, psicología, ciencias de la gestión, medio ambiente, etc. está aumentando la cifra de tales publicaciones; en ellas se han popularizado acrónimos tales como LULU (locally unwanted land uses), NIMBY (not in my back yard) o NIABY (not in anybody back yard) para referirse a esos síndromes o conflictos 
socio-políticos bien conocidos.

El análisis espacial del bienestar o malestar sentido por los ciudadanos ante actividades molestas, insalubres o peligrosas cercanas 0 ante riesgos naturales cuenta con una tradición importante en Geografía (vid. Golledge y Stimpson, 1997) y en Psicología Ambiental (Jiménez Burillo y Aragonés, 1991; Valera et al., s.f.). Procedimentalmente el recurso a instrumentos de encuesta ha sido frecuente para determinar la percepción y actitudes hacia esos problemas ambientales y los efectos vividos (e.g. tipo, intensidad, duración, etc.) mediante escalas ordinales (e.g. Bale, 1981, Schroeder, 1984). Diversos trabajos han abordado así la determinación de los conocidos como campos de externalidades sentidas para actividades de servicios tales como hospitales psiquiátricos (Dear et al. 1980), campos de fútbol (Mason y Robins, 1991; Mason y Moncrieff, 1993), centros de atención a drogodependientes (Moreno, 1992 y 1998), auditorios de conciertos al aire libre (Chase y Healey, 1995) y parques públicos (Pérez-Tejera, 2012). Igualmente, para actividades económicas como las industriales, Ford et al. (2007) acometieron un estudio para evaluar el entorno de las escuelas, plasmando cartográficamente los lugares (puntos) y recorridos evaluados por los niños encuestados, lo que permitió aprehender diversos atributos percibidos de ese entorno; y Sofer et al. (2012) describen el impacto en la calidad de vida de la población cercana a pequeñas industrias y talleres, producto de molestias por ruido, emisiones al aire y contaminación del suelo. Indagaciones específicas sobre la percepción de la polución aérea son también abundantes, como evidencia la revisión de Catalán-Vázquez y Jarillo-Soto (2010) y en ellas se han documentado hallazgos valiosos para interpretar y explicar relaciones con otras variables socioeconómicas, funcionales o espaciales.

En esta contribución se busca desvelar aspectos relevantes de la geografía de los perjuicios percibidos e imputados subjetivamente a una actividad industrial cercana. De forma concreta se pretende:

- Adoptar una metodología geográfica para medir y visualizar la cuantía del malestar social, mediante mapas novedosos mostrando dónde y cuánto se sienten los efectos negativos.

- Caracterizar los patrones espaciales del malestar socio-ambiental, desagregando por tipos de efectos distintos.

- Ensayar la elaboración de mapas sintéticos de multiafección percibida, a partir de los patrones parciales. 
Con ello se pretende mostrar un procedimiento para mejorar los diagnósticos acerca de los impactos de actividades humanas, centrado específicamente en la dimensión subjetiva de dichos efectos, es decir, en el malestar percibido por los ciudadanos el cual, en tanto que externalidad espacial elusiva, pero real y medible, pueda ser interpretado y valorado más rigurosamente por parte de los agentes sociales y los decisores públicos y privados, a efectos de eventuales medidas de planificación o gestión.

\section{MATERIALES Y MÉTODOS}

\subsection{Localización del área de estudio}

El ámbito elegido se sitúa en el borde costero de dos municipios de la parte central de Chile: Quintero y Puchuncaví (región de Valparaíso). El Decreto Supremo №346 del Ministerio de Agricultura chileno del año 1993 declara que la zona saturada por anhídrido sulfuroso (SO2) y material particulado respirable (MP10) es aquella circundante al gran Complejo Industrial Ventanas, que se ubica jurisdiccionalmente en las comunas de Quintero y Puchuncaví. Entre las coordenadas UTM Zona 19 H: 6387381,3 - 6370657,6 Norte y 262413,3 - 286472,9 Este.

Los límites que se establecen en el Decreto son los siguientes:

- Norte: desde el océano Pacífico hasta la desembocadura del Estero Catapilco, y siguiendo este hasta su confluencia con el Estero La Canela.

- Sur: desde la cumbre del Cerro Colorado, bajando en línea recta hasta la intersección del Canal Mauco con el Estero Mantagua.

- Este: desde la cumbre Cerro Alto del Agua del Peumo, siguiendo por la divisoria de aguas hasta el cerro Pucalán, continuando en línea recta hasta la cumbre del Cerro Piedra Trepada.

- Oeste: línea de costa del océano Pacífico, desde la desembocadura del Estero Mantagua por el sur, hasta la desembocadura del estero Catapilco por el norte.

La superficie es de 402,36 km2 y administrativamente limita con las comunas de Nogales al este, Concón al sur, Zapallar al norte y con el océano Pacífico al oeste.

Para este caso, el área de estudio se ha acotado a los centros poblados que se ubican al interior de las citadas comunas: Quintero, Loncura, Ventanas, La Greda, La Chocota, Horcón y Puchuncaví (véase figura 1). En ella se observa la existencia de varios asentamientos, ubicados a distinta distancia (desde los ad- 
yacentes hasta los sitos a unos $7 \mathrm{~km}$ aproximadamente) y orientación respecto al complejo industrial, y conteniendo cifras de población diferentes. Ello les expone a los efectos ambientales externos de las fábricas de una manera clara.

Figura 1: La zona de estudio, sita entre las comunas de Quintero y Puchuncaví, Región de Valparaíso, Chile

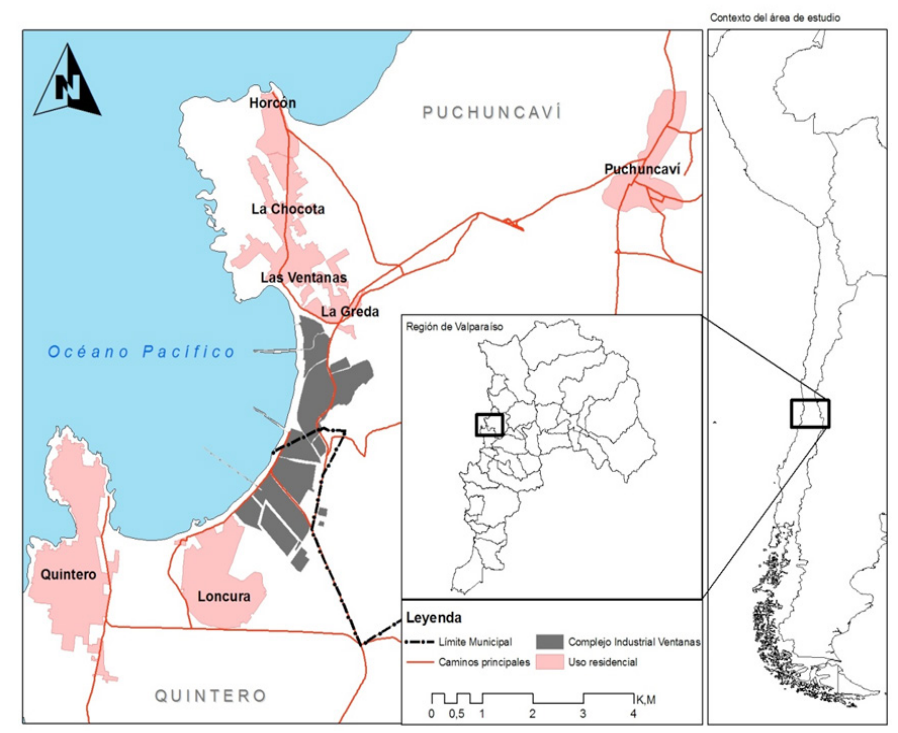

Fuente: Elaboración propia.

\subsection{Fuentes de información}

\subsubsection{Encuestas a la población residente}

El objetivo de la aplicación del instrumento fue evaluar la percepción de los efectos ambientales sobre la población y el territorio, considerando el nivel de intensidad global y la variabilidad temporal de las molestias sentidas en un período dado (el último año) por el encuestado y por otros miembros del hogar, debido a la polución industrial.

La selección de los encuestados se basó en un muestreo espacial sistemático, considerando como universo a un representante por vivienda. La selección de las viviendas se sustentó en recorridos exhaustivos de todos los frentes de manzana con viviendas en la zona asignada a cada encuestador. Los equipos de 
trabajo de campo se desplegaron en las siguientes unidades territoriales: Quintero, Loncura, La Greda, Ventanas, La Chocota, Horcón y Puchuncaví, obteniéndose 290 encuestas para todo el ámbito en estudio. La encuesta se realizó mediante entrevistas domiciliarias durante el mes de marzo de 2012. En ellas se requirió a los encuestados pronunciarse acerca de si en el último año habían percibido ciertos tipos de efectos (malos olores, ruidos, polución atmosférica, etc.) procedentes del complejo industrial, su intensidad y frecuencia (aunque estos dos últimos aspectos no serán analizados aquí, por limitaciones de espacio).

\subsubsection{Otras fuentes}

Junto a los datos de la encuesta se ha utilizado cartografía digital sobre los asentamientos, límites municipales, red viaria, etc. procedente del Sistema Integrado de Información Territorial (SIIT) de la Biblioteca del Congreso Nacional de Chile.

\subsection{Técnicas de análisis}

Puesto que los efectivos de la muestra en cada asentamiento son aproximadamente proporcionales a la población, la teoría del muestreo permite asumir una aceptable representatividad de la misma en su cuantía total y en las respuestas, lo que equivale a decir, a su percepción de los problemas ambientales. A la hora, por tanto, de analizar espacialmente los resultados es sostenible adscribir tales respuestas al domicilio de los encuestados y así se hizo al georreferenciar los datos de la encuesta. En principio, cabe pensar en varias alternativas para representar cartográficamente y analizar las respuestas cualitativas sobre la percepción individual de los efectos molestos. Tras algunos ensayos, pareció más prometedor adoptar el planteamiento y técnica utilizados por Moreno (1992 y 1998). En síntesis, se parte de asumir una clasificación previa de las respuestas a cada pregunta y retener particularmente las que afirman la percepción de molestias, por cuanto son expresión del malestar asociado a una externalidad concreta. La trascripción espacial y visualización de dichas respuestas bien podría recurrir a una cartografía de símbolos puntuales bajo el modelo vectorial de los SIG, pero para una representación más realista de la espacialidad humana y una mejor aprehensión de los resultados, pareció más acertado recurrir a un estimador de densidad núcleo o kernel y elaborar mapas bajo el modelo raster. Su aplicación implica asumir, de acuerdo con la lógica de los estimadores kernel" que el dato puntual en cuestión se despliega por un entorno alrededor de la vivienda, de tamaño dado por la "anchura de la ventana" (bandwidth o search radius) del kernel. Ello resulta congruente con un en-

\footnotetext{
${ }^{1}$ Una presentación de tales estimadores puede verse en diversas obras, por ejemplo, Moreno (1991), Santos Preciado y García Lázaro (2008: 267-312).
} 
tendimiento de la espacialidad del individuo, que aunque centrada en su domicilio, en su movilidad espacio-temporal habitual se despliega por el entorno del mismo y probablemente con una frecuencia (densidad) decreciente desde el punto-domicilio hasta una distancia razonable (definida por la anchura de la ventana). Se trataría de una especie de "entorno personal próximo" (EPP) en el que la persona desarrolla actividades cotidianas varias, centradas y factibles desde el punto-domicilio con coste casi irrelevante. A efectos de nuestro análisis pareció conveniente fijar ese parámetro en $500 \mathrm{~m}$, lo cual significa que las densidades conllevan un reparto del peso unitario de cada respuesta afirmativa en un círculo de tal radio, aunque de manera desigual según la lógica del kernel: más intenso en la parte central y más somero en la periferia del círculo. Según este planteamiento, se obtendrán en la capas raster resultantes de cada pregunta píxeles con mayor densidad allí donde se concentran las respuestas afirmativas y con menor densidad donde hay pocas de tales respuestas. Se tendría por tanto una plasmación cuantitativa del malestar socio-ambiental sentido y desvelado espacialmente a través de la muestra. Ahora puede comprenderse la importancia de lograr en el muestreo una distribución espacial de la muestra bien representativa (v.gr. con un procedimiento sistemático, como aquí se hizo). Un sesgo en ello implicaría la invalidez (por ese defecto) del tratamiento analítico que hemos descrito. Procede añadir que el tamaño del píxel adoptado es de $50 \mathrm{~m}$, con el criterio de obtener capas raster sin la perturbación visual del pixelado.

Las capas de densidad en cuestión se representan con una paleta monocroma consistente sin intervalos, para evitar la rigidez asociada a ellos. Se adopta pues una gradación continua de color en correspondencia lineal con la amplitud de los datos, mediante lo que se conoce como expansión lineal del contraste o linear stretch (Minimum-Maximum). Ahora bien, dado que la amplitud de las densidades de cada capa suele diferir, pues el valor máximo depende de la frecuencia y ubicación de las respuestas afirmativas a cada pregunta, se ha evitado la inconsistencia entre capas adoptando (forzando) una amplitud constante para la paleta de color: la de la capa de densidades de la muestra total de encuestados, que representaría la situación de densidad máxima (por incluir a la totalidad de los $n$ residentes encuestados). Ello implicará que, a menudo, en los mapas de cada uno de los efectos percibidos, la totalidad de la paleta cromática no llegue a mostrarse; en particular, los tonos más intensos asignados a los valores altos probablemente no aparecerán. Esto, en lugar de ser un inconveniente, resulta una ventaja, pues asegura la comparabilidad de los mapas, mediante una "normalización" de la paleta, que hace innecesaria la normalización de los datos de densidad a los efectos de percepción visual. 
Adicionalmente, y puesto que la gradación continua de color no propicia una aprehensión sintética y simple, se ha introducido otro componente cartográfico: las isolíneas a intervalos regulares de 0,1 encuestados / ha, las cuales permiten resaltar con nitidez las zonas donde se concentran los encuestados que reconocen una molestia ambiental concreta. Su gradación en grosor y las etiquetas cuantitativas con los valores de iso-molestias completan las ayudas a la apreciación.

Los tratamientos han sido desarrollados mediante ArcGIS, programa en el que la función disponible de densidad núcleo (kernel) para un lugar (píxel) dado, s, es la cuadrática descrita en Silverman (1986, p. 76, ecuación 4.5):

$$
f^{\prime}(s)=\sum_{d_{i_{s}} h} \frac{3}{\pi h^{2}}\left(1-\frac{d^{2}}{h^{2}}\right)^{2}
$$

Siendo $d_{i}=$ la distancia entre el punto i y el lugar $s, y ~ h=$ anchura de la ventana de búsqueda.

Como información comparativa relevante se presentarán también los estadísticos descriptivos básicos de cada capa.

Con objeto de disponer de una visión sintética de los efectos negativos percibidos se ha construido además un índice sencillo que trata de resumir los resultados de las cinco preguntas según una lógica aditiva. A tal fin, para cada encuestado se obtuvo la proporción del número de respuestas afirmativas respecto al total de efectos considerados (5) y sobre tal valor fue aplicado el estimador kernel.

\section{ANÁLISIS DE RESULTADOS}

\subsection{La distribución por localidades de la muestra y de los problemas ambientales percibidos}

Antes de analizar los patrones de densidad de la afección ambiental sentida conviene presentar sucintamente la distribución espacial de la muestra y de las respuestas generales para una adecuada contextualización de las mismas. En la Tabla 1 se constata que el reparto de los encuestados es desigual, en correspondencia con el tamaño de las localidades. El conjunto del área de estudio exhibe también porcentajes diferentes según el tipo de afección: la contaminación del aire es muy altamente sentida (76,90\% de los encuestados), seguida de los olores $(61,72 \%)$. La suciedad en viviendas y el ruido se sitúan en tercer lugar con unas 
frecuencias ligeramente superiores a la mitad de la muestra $(54,83$ y $52,76 \%)$ y finalmente la conciencia de problemas de salud es algo menos referida $(39,31 \%)$. Aunque tentativamente, cabría interpretar estos datos como una manifestación de la prevalencia de las sensaciones visuales (humos y suciedad), olfativas y auditivas, por este orden, en tanto que los efectos sobre la salud, aunque pudiesen ser más lesivos, quedan bastante rezagados en la percepción social, quizá por la dificultad de establecer subjetivamente la conexión causal con las emisiones industriales. En todo caso, los niveles de conciencia individual de los problemas son importantes, pues en todos los efectos, excepto uno, se supera la mitad de los encuestados.

En el tabla 1 es posible apreciar igualmente el grado de conciencia de los efectos en las distintas localidades. Al margen de las diferencias absolutas entre ellas, imputables en parte a la proporcionalidad de las submuestras, lo más revelador estriba en que, en ciertos lugares, los porcentajes de afección sentida exceden de manera notoria a los de la submuestra respectiva (primera fila de la tabla). Se han resaltado las celdas con un exceso de dos puntos porcentuales, lo que permite constatar que los asentamientos de Loncura y Horcón-Las Ventanas-La Greda exhiben una hipersensibilidad clara. Su posición más próxima al complejo industrial que los otros lugares aparece como la causa más verosímil de ese resultado.

Tabla 1: Distribución por localidades de la muestra y de la afección percibida por los encuestados

\begin{tabular}{|c|c|c|c|c|c|c|c|c|c|c|}
\hline LOCALDADES & Total & $\%$ & Quintero & $\%$ & Loncura & $\%$ & $\begin{array}{c}\text { Las Venta- } \\
\text { nas - Horcón }\end{array}$ & $\%$ & $\begin{array}{c}\text { Puchunca } \\
\text { ví }\end{array}$ & $\%$ \\
\hline $\mathrm{N}^{0}$ Encuestados & 290 & 100 & 72 & 24.83 & 47 & 16.21 & 117 & 40.34 & 54 & 18.62 \\
\hline $\begin{array}{l}\text { TIPO DE } \\
\text { AFECCION }\end{array}$ & SI (frec) & $\%$ & SI (frec.) & $\%$ & SI (frec) & $\%$ & SI (frec) & $\%$ & SI (frec) & $\%$ \\
\hline Olor & 179 & 100 & 40 & 22.35 & 29 & 16.20 & 74 & 41.34 & 36 & 20.11 \\
\hline Ruido & 153 & 100 & 22 & 1438 & 28 & 18.30 & 88 & 57.52 & 15 & 9.80 \\
\hline $\begin{array}{l}\text { Contaminación del } \\
\text { aire }\end{array}$ & 223 & 100 & 50 & 22.42 & 30 & 13.45 & 96 & 43.05 & 47 & 21.08 \\
\hline Problemsas de sahd & 114 & 100 & 21 & 18.42 & 28 & 24.56 & 43 & 37.72 & 22 & 19.30 \\
\hline $\begin{array}{l}\text { Suciedad en } \\
\text { vivienda }\end{array}$ & 159 & 100 & 33 & 20.75 & 28 & 17.61 & 71 & 44.65 & 27 & 16.98 \\
\hline
\end{tabular}

Nota: Porcentajes horizontales.Celdas en rosa: porcentajes $>2$ puntos al de la muestra (primera fila). Fuente. Elaboración propia.

\subsection{La densidad espacial de la muestra}

Como segundo punto se describirán los patrones conformados por las densidades espaciales de los encuestados. En primer lugar se mostrará la densidad kernel de la muestra, a efectos de tomarla como referencia para el análisis de los efectos 
ambientales que los residentes han percibido y una caracterización estadística sumaria del patrón de densidad de cada efecto.

En el mapa (Figura 2) se aprecia que las mayores densidades de encuestados aparecen en el ámbito de Las Ventanas-Horcón y Punchucaví. En Quintero son inferiores, aunque con un ligero aumento en su parte sur, donde en la última década se ha consolidado la zona de expansión urbana. En Loncura y La Greda se alcanzan densidades más bajas, en consonancia con el tipo de hábitat existente. En la Tabla 2 se recogen los resúmenes estadísticos de las capas raster de densidad obtenidas para los distintos efectos investigados. Ciertos estadísticos exhiben valores acordes con la frecuencia de las respuestas, por lo tanto en línea con lo esperado: así los valores máximos y las medias poseen un notable paralelismo (los mínimos no son relevantes, pues tienden siempre a cero, por efecto matemático del estimador). El coeficiente de variación, sin embargo, desvela que en tres de los efectos (olores, ruidos y polución atmosférica) las desigualdades relativas en las densidades son algo mayores que incluso las de la muestra total. Ello implicaría que espacialmente las divergencias sociales al respecto crecen, lo que podría interpretarse como una polarización relativamente mayor de las actitudes en tales casos.

Figura 2: Densidad núcleo (kernel) de los encuestados en el área próxima al complejo Ventanas, Chile

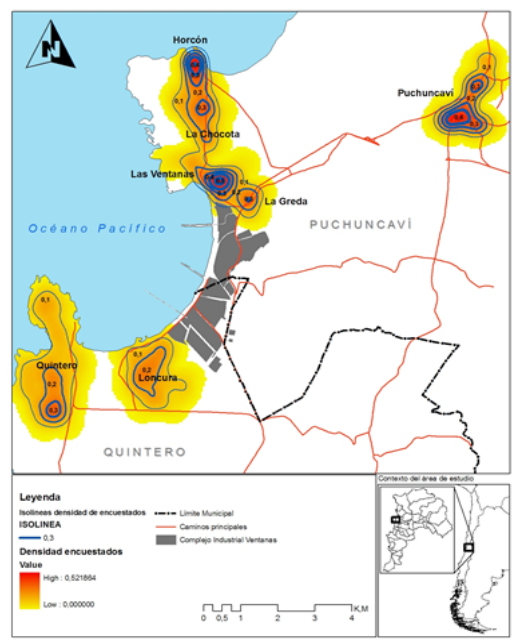

Fuente: Elaboración propia. 
Tabla 2: Estadísticos de las capas de densidades focales (núcleo o kernel) de los encuestados totales y de los que han percibido efectos ambientales en el entorno del complejo industrial Ventanas, Chile. Unidades: Hab. por 0,25 ha.

\begin{tabular}{|c|c|c|c|c|c|c|}
\hline & & \multicolumn{5}{|c|}{ TIPO DE EFECTO PERCIBIDO } \\
\hline $\begin{array}{c}\text { ESTADÍSTI- } \\
\text { CO }\end{array}$ & $\begin{array}{c}\text { TOTAL } \\
\text { MUESTRA }\end{array}$ & OLORES & RUIDOS & $\begin{array}{c}\text { POLU- } \\
\text { CION EN } \\
\text { AIRE }\end{array}$ & $\begin{array}{c}\text { PROBLEMAS } \\
\text { DE SALUD }\end{array}$ & $\begin{array}{c}\text { DAD EN } \\
\text { VIVIEN- } \\
\text { DA }\end{array}$ \\
\hline MEDIA & 0,112 & 0,073 & 0,068 & 0,089 & 0,052 & 0,067 \\
\hline $\begin{array}{c}\text { DESVIA- } \\
\text { CIÓN TÍPI- } \\
\text { CA }\end{array}$ & 0,103 & 0,071 & 0,067 & 0,085 & 0,047 & 0,062 \\
\hline $\begin{array}{c}\text { COEF. DE } \\
\text { VARIACIÓN }\end{array}$ & 0,920 & 0,973 & 0,991 & 0,960 & 0,916 & 0,918 \\
\hline MÁXIMO & 0,522 & 0,396 & 0,365 & 0,474 & 0,236 & 0,326 \\
\hline MÍNIMO & 0,002 & 0,002 & 0,001 & 0,002 & 0,001 & 0,001 \\
\hline
\end{tabular}

Nota: Se han excluido de los cálculos los valores nulos.

Fuente. Elaboración propia.

\subsection{El malestar sentido según tipos de efectos ambientales}

\subsubsection{La percepción de olores}

La percepción vecinal de olores (Figura 3) evidencia unas densidades mayores en las localidades Las Ventanas y Horcón (extremo N), seguidas de Puchuncaví (NE). Si bien la primera está relativamente cercana a las industrias, los otros dos asentamientos quedan más distantes. En Loncura (adyacente a la zona industrial) y Quintero el impacto resulta menos densamente percibido (solo valores bajos y medio bajos de densidad), pese a la superior proximidad al foco contaminante valorado. Estos resultados cabría relacionarlos con la dirección del viento predominante que en el periodo diurno es de suroeste, mientras que en el periodo nocturno es del Este. 


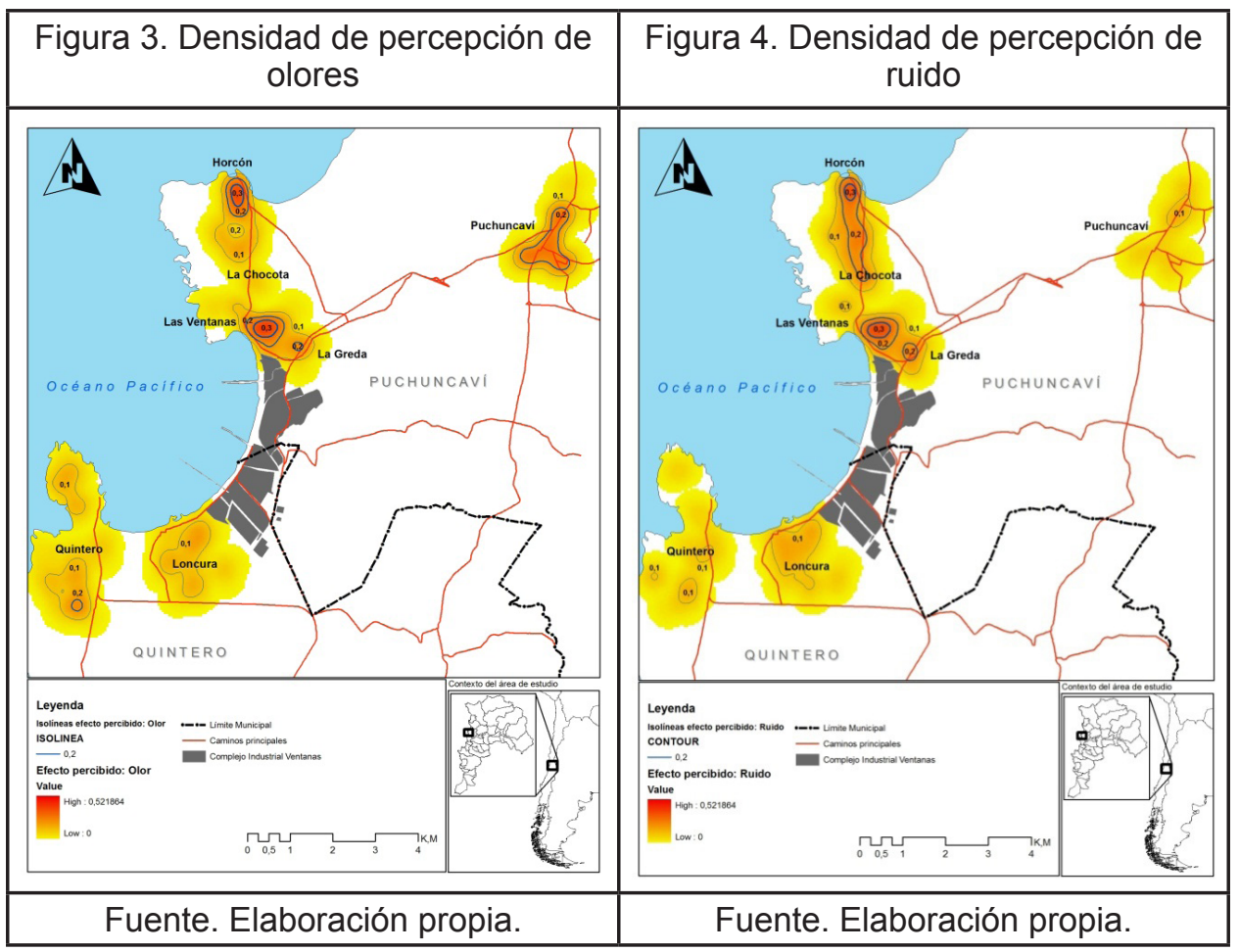

\subsubsection{La percepción de ruidos}

En el caso del ruido (Figura 4) la percepción ciudadana exhibe espacialmente cambios significativos en su densidad, sobre todo cuando se la compara con la de muestra total. Aparece una mayor y amplia sensibilidad en Horcón - Las Ventanas; allí el área con densidad de afectados media-alta aparece más extendida que para los olores. Ello refleja también el hecho de que la cifra de afectados (Tabla 1) sea proporcionalmente superior. Por el contrario, las densidades (y los porcentajes de la Tabla 1) bajan ostensiblemente en Puchuncaví y Quintero. Recordando los factores incidentes en la difusión espacial de las ondas sonoras, entre ellos el descenso bastante rápido con la distancia: $6 \mathrm{~dB}$ de reducción al doblarse la distancia (Warring, 1983: 8), y el mayor alejamiento de las dos localidades mencionadas respecto al complejo fabril, resultan coherentes esas disminuciones en las apreciaciones subjetivas. En Loncura, localidad con un número de afectados proporcionalmente alto, no aparecen sin embargo concentraciones relevantes en las densidades de afección, aunque el patrón de su zona más afectada (desplazada hacia el norte) sea algo distinto al de los olores. 


\subsubsection{La percepción de contaminación atmosférica}

La contaminación atmosférica es, como se dijo antes, el efecto más constatado por los residentes. El mapa de percepción (Figura 5) denota densidades elevadas en Horcón-Las Ventanas y Puchuncaví, con un notable paralelismo al de la distribución muestral. La afección más destacada en tales localidades (sitas al $\mathrm{N}$ y NE del complejo industrial) habrá de ser puesta en relación con factores como los vientos diurnos dominantes. En Loncura y Quintero (parte sur) la extensión de la mancha de densidades medias crece también respecto a otros efectos, si bien proporcionalmente no destacan. Ambos casos se ubican al W de la zona industrial, lo que habrá de ser explorado con datos complementarios.

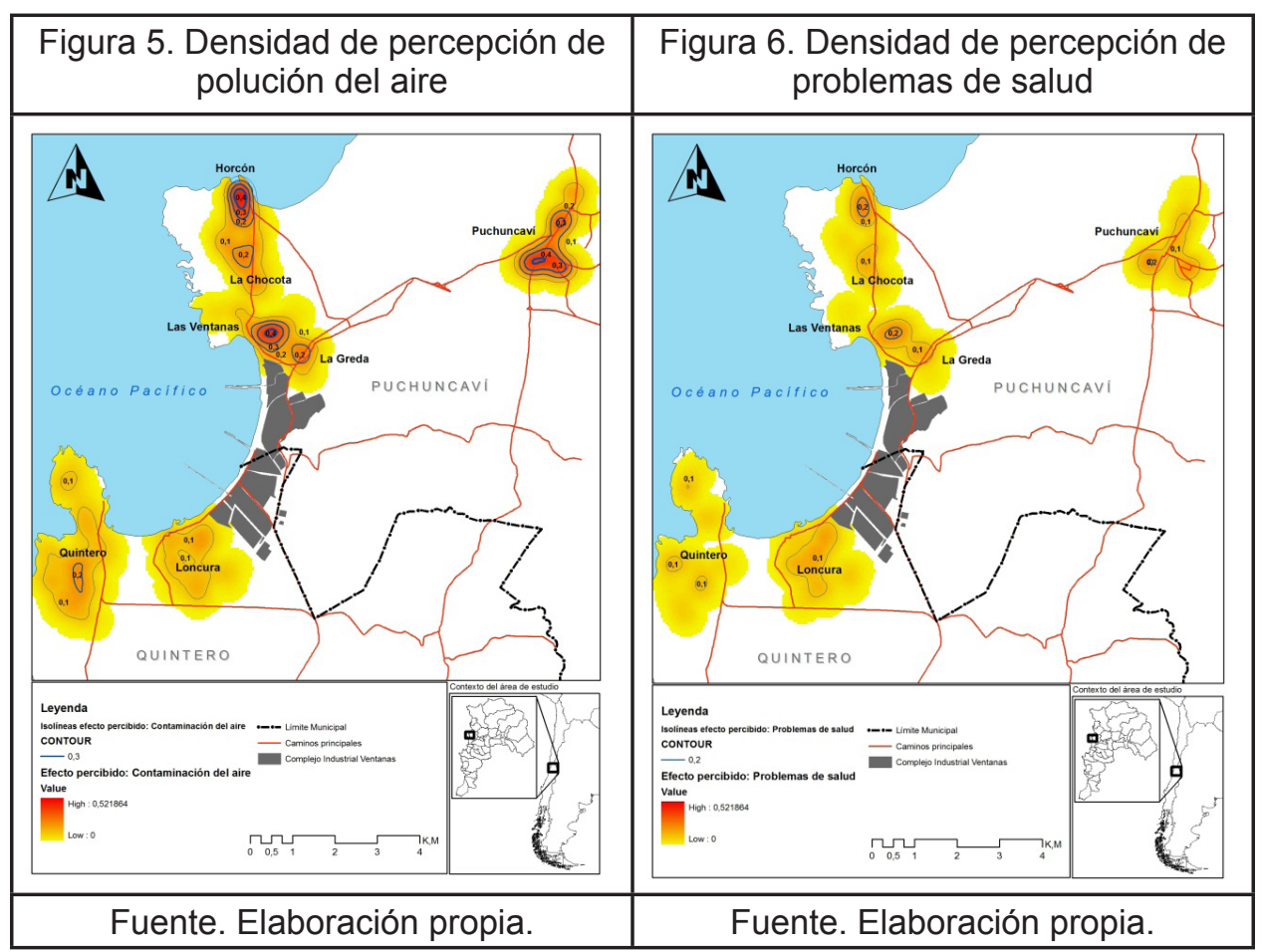

\subsubsection{Los problemas de salud percibidos}

La asociación entre morbilidad y emisiones industriales resulta débil en la mente de los residentes, como mostró su porcentaje medio-bajo. Ello se plasma cartográficamente en unos niveles de densidad medios y bajos en casi todas las localidades examinadas (Figura 6). Quizá el hecho más destacable sea la persistente extensión de la zona de densidad media en Loncura. Esto puede relacionarse con 
su contigüidad al complejo industrial y con la existencia de una cifra de afectados proporcionalmente superior, tal como se evidenció en el Cuadro 1.

\subsubsection{La suciedad en viviendas}

Este efecto, sentido por algo más de la mitad de los encuestados, se manifiesta proporcionalmente más solo en Horcón - La Ventanas, siendo la densidad mayor en la zona concreta de La Greda, adyacente al complejo (Figura 7). En los restantes lugares las densidades predominantes son medio-bajas y bajas.

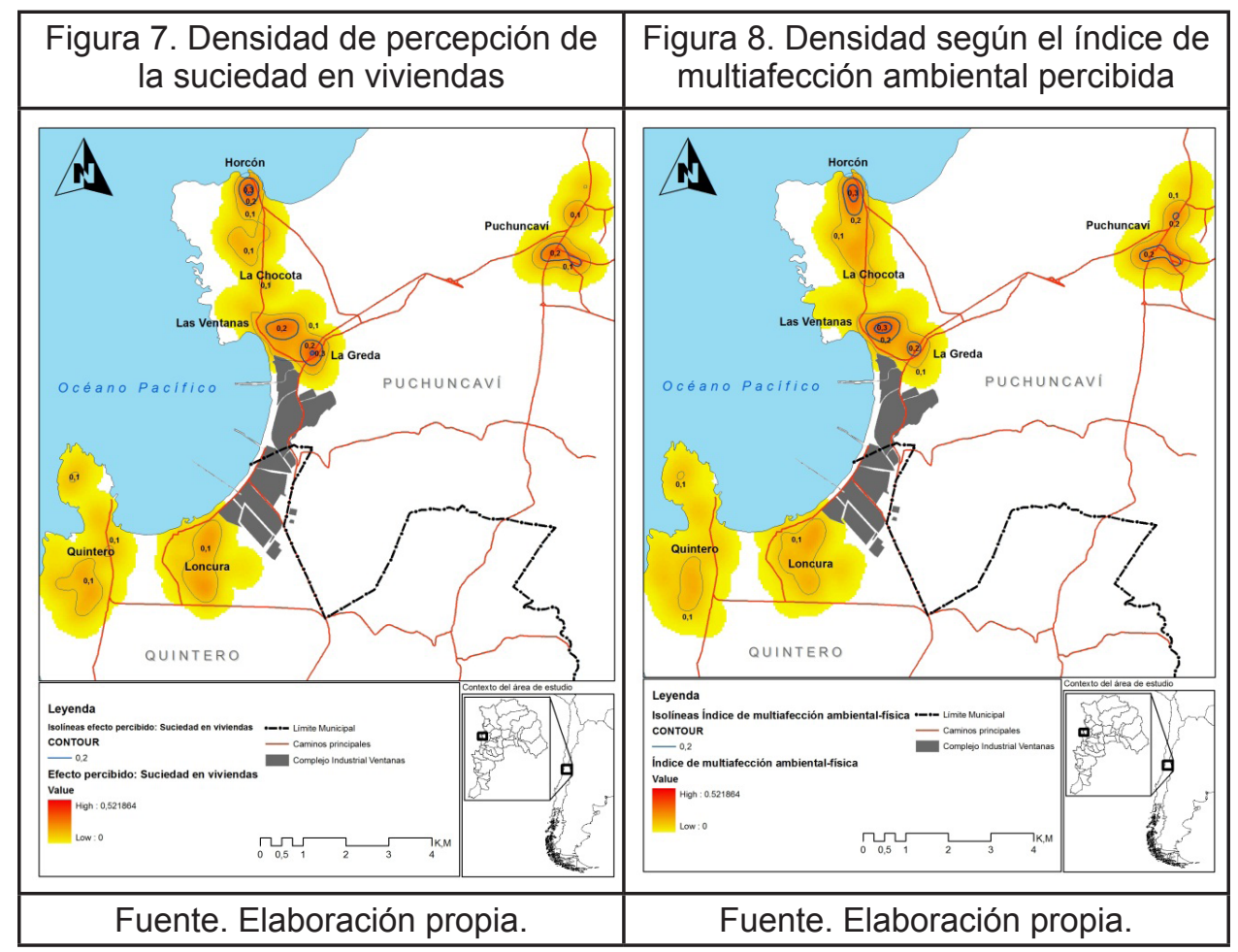

\subsubsection{Diagnóstico sintético de la multiafección ambiental percibida (IMAP)}

El último mapa elaborado (Figura 8) intenta resumir mediante un índice de forma aditiva las respuestas a las cinco preguntas directas sobre la percepción social de los problemas ambientales. En conjunto, niveles medio-altos solo afloran en algunas zonas pequeñas de Horcón - Las Ventanas sitas al norte y un poco en el sur de Puchuncaví. En este asentamiento prevalecen las densidades bajas y medio-bajas de afectados, siendo esa la tónica ahora también de Loncura y Quin- 
tero. En el primero de ellos la zona con densidad medio-baja sigue exhibiendo una extensión muy notable respecto al área del asentamiento, rasgo que ha persistido a lo largo de los mapas expuestos.

\section{DISCUSIÓN Y CONCLUSIONES}

Desde una perspectiva metodológica, en otros trabajos similares sobre efectos ambientales de actividades humanas el acento se ha puesto en obtener datos estadísticos por unidades espaciales (e.g. asentamientos) que pueden resultar grandes y a veces vagamente definidas (e.g. Sofer et al., 2012), o en determinar campos de externalidades subjetivas a partir de una apreciación de la intensidad del efecto percibido mediante una escala ordinal (e.g. Bale, 1981 y 1990; Mason y Robins, 1991; Mason y Moncrieff, 1993; Chase y Healey, 1995). Esto último posibilita plasmar en mapas de isopletas dicha intensidad. Su significado sería equivalente al de los niveles de inmisión estimados para contaminantes atmosféricos.

Sin embargo, tales mapas no reflejan, ni permiten conocer la densidad del malestar social en cada lugar, pues esta depende de la distribución espacial del universo y de la muestra. Si ésta se seleccionó espacialmente de forma representativa, entonces las respuestas contienen, además del nivel de molestia (si se averiguó en la encuesta), un significado cuantitativo adicional, por cuanto la cifra total de los afectados expresaría la magnitud del impacto negativo absoluto, lo que sería asimilable al malestar total recogido en la muestra.

A diferencia de otros estudios previos que eluden tal aspecto, en el presente trabajo el foco se ha centrado en la "espacialización" de ese malestar total. Frente a la alternativa de resumir los hallazgos por localidades, aquí se ha propuesto una aproximación muy desagregada espacialmente, basada en la metodología planteada por Moreno (1992 y 1998), en aras de dar una visión más precisa de los impactos socio-ambientales allí donde ocurren. Con tales resultados se desvela la distribución espacial del malestar social imputable subjetivamente a las externalidades ambientales generadas por el complejo industrial. En efecto, si las cifras y porcentajes de respuestas afirmando esa afección traducen la magnitud de la misma y si se posee su referencia espacial, es factible entonces usar un procedimiento como el estimador núcleo (kernel) para plasmarla y examinarla espacialmente de forma detallada. Procede recordar que, por la estructura matemática de las funciones de densidad kernel, el valor unitario de cada afectado se reparte (según el tipo de kernel) entre los píxeles del entorno del punto-domicilio de manera descendente con la distancia, pero manteniendo la propiedad de que el volumen bajo la superficie de densidad es equivalente a la frecuencia de los puntos, i.e. a la cantidad de 
afectados y a lo que representan, el malestar total capturado por la muestra. Ello hace posible avistar operaciones con SIG para analizar espacialmente esas distribuciones y explorar nuevas relaciones, cuestiones que aquí no se han abordado. En síntesis, uno de los resultados más destacables de este trabajo reside en haber mostrado cómo los datos muestrales pueden ser traspuestos a valores en las capas raster de densidad de afectados, que constituyen una estimación aproximada de la cantidad de malestar sentido en cada lugar (y con la resolución espacial que se desee).

Desde el punto de vista empírico, esta investigación ha permitido conocer una variedad de patrones de malestar socio-espacial subjetivo asociados a un gran complejo industrial. En ellos se han desvelado diferencias espaciales vinculables, por un lado, a las propias densidades espaciales de la muestra (proporcionales a la del universo), lo cual simplemente refleja que la mayor concentración de residentes tiende a ocasionar concentraciones de malestar, aunque no siempre en una cuantía proporcional; por otro, se han observado variaciones que podrían estar relacionadas con la distancia al complejo industrial o con la posición relativa (orientación), probablemente, como consecuencia de la forma de dispersión de los contaminantes. Algunos de estos hallazgos tienen cierta analogía con los descritos en Moreno (1998). En todo caso, relaciones como las recién mencionadas no se han podido investigar aquí y se abordarán en ulteriores análisis. Igualmente, otras cuestiones atingentes a los correlatos de las respuestas con los atributos personales o su asociación con algunos indicadores objetivos (mediciones de las inmisiones con instrumentos ad hoc), para fines explicativos quedan pendientes de explorar.

\section{BIBLIOGRAFÍA}

- Bale, J. (1981). "Teaching welfare issues in Geography", en Walford, R. (ed.): Signposts for geography teaching. Londres, Longman, p. 51-63.

- Bale, J. (1990): "In the shadow of the stadium: football grounds as urban nuisances", en Geography, 75, 325-344.

- Barroto, M., Rodríguez, L., Reyes, A. y López, B. (2011). "Percepción ambiental en dos comunidades cubanas", en M+A Revista electrónica de Medio Ambiente, 10, 13-29. Disponible en http://revistas.ucm.es/index.php/MARE/issue/view/ MARE11111/

- Buzai, G. D. (2011). "La geotecnología: ¿Nuevo paradigma de la geografía o paradigma geográfico de la ciencia?", Revista Catalana de Geografía, XVI, 42, 
10 pp. www.rcg.cat/articles.php?id=187

- Buzai, G. (2011). "La construcción de mapas mentales mediante apoyo geoinformático. Desde las imágenes mentales perceptivas hacia la modelización digital", en Revista Geográfica de Valparaíso, 44, pp. 1-17. Disponible en http:// www.rgv.ucv.cl/Articulo44_1.pdf

- Catalán-Vazquez, M. y Jarillo-Soto, E. (2010). "Paradigmas de investigación aplicados al estudio de la percepción pública de la contaminación del aire", en Rev. Int. Contam. Ambient. 26 (2), pp. 165-178.

- Catling, S.J. (1978). "Cognitive mapping exercises as a primary geographical experience", en Teaching Geography, 3, p. 120-123.

- Chase, J. y Healey, M. (1995). "The spatial externality effects of football matches and rock concerts - The case of Portman Road Stadium, Ipswich, Suffolk", en Applied Geography, 15, 1, p. 18-34.

- Dear, M. Taylor, S. M. y Hall, G. B. (1980). "External effects of mental health facilities", en Annals of the Association of American Geographers, 70, 3, p. 342352.

- Ford, S., Moore, J., Niswonger, J. y Walsh, E. (2007). "GIS technical report: Spatial distribution of industrial land use and children's perception of safety and their environment". The University of Texas at Austin. Disponible en http://soa. utexas.edu/work/eaejp/Papers/GIS\%20Report.pdf

- Golledge, R. y Stimpson, R. (1997). Spatial behavior. A geographic perspective. New York: The Guilford Press.

- Gould, P. (1975). People in information space: The mental maps and information surface of Sweden. Lund Studies in Geography, The Royal University of Lund/CWK Gleerup.

- Gould, P. y White, R. (1974) Mental maps. Londres: Penguin. 
- Jiménez Burillo, F. y Aragonés, J. I. (1991). Introducción a la Psicología Ambiental. Madrid: Alianza Editorial.

- Lynch, K. (1960). The image of the city. Cambridge, Massachusetts: MIT Press.

- Mason, C. y Moncrieff, A. (1993). "The effect of relocation on the externality fields of football stadia: The case of St Johnstone FC", en Scottish Geographical Magazine, 109, 2, p. 96-105.

- Mason, C. y Robins, R. (1991). "The spatial externality fields of football stadiums: the effects of football and non-football uses at Kenilworth Road, Luton", en Applied Geography 11(4):251-266.

- Moreno Jiménez, A. (1991). "Modelización cartográfica de densidades mediante estimadores Kernel", en Treballs de la Societat Catalana de Geografía, 30, p. 155-170. Disponible en http://www.raco.cat/index.php/TreballsSCGeografia/ article/view/184519/237797

- Moreno Jiménez, A. (1992). "Spatial impact analysis and site selection for controversial public facilities with GIS". EGIS'92, en Third European Conference and Exhibition on Geographical Information Systems.Utrecht, EGIS Foundation, vol. 1, p. 483-491.

- Moreno Jiménez, A. (1998). "Los mapas de actitudes hacia el entorno: un instrumento para la exploración del espacio psico-social y la toma de decisiones con S.I.G.", en Alegre, P. et al. (Coord.): Tecnología geográfica para el siglo XXI. VIII Coloquio de Geografía Cuantitativa. Barcelona, Universidad Autónoma de Barcelona, p. 247-263. Disponible en http://age.ieg.csic.es/metodos/ barcelona98/1998_018_moreno.pdf

- Moreno Jiménez, A. (2010). "GeoFocus: diez años en el camino hacia la sociedad de la geoinformación", en GeoFocus (Editorial), 10, pp. 1-6.

- Moreno Jiménez, A. (2013). "Entendimiento y naturaleza de la cientificidad geotecnológica: una aproximación desde el pragmatismo epistemológico", en Investigaciones Geográficas (en evaluación).

- Moreno, A. y Escolano, S. (1992). El comercio y los servicios para la produc- 
ción y el consumo. Madrid: Síntesis.

- Pérez-Tejera, F. (2012)."Diferencias entre los usuarios de seis parques públicos en Barcelona según el nivel de seguridad percibida en el barrio", en Athenea Digital, 12 (1), p. 55-66.

- Pinch, S. (1985). Cities and services. The geography of collective consumption. Londres: Routledge and Kegan Paul.

- Santos Preciado, J. M. y García Lázaro, F. J. (2008). Análisis estadístico de la información geográfica. Madrid: UNED

- Schroeder, H. (1984). "Environmental perception rating scales. A case for simple methods analysis", en Environment and Behavior, 16, 5, p. 573-598.

- Silverman, B. W. (1986). Density estimation for statistics and data analysis. New York: Chapman and Hall.

- Sofer, M., Potchter, O., Gnaim, N. y Gnaim, J. (2012)."Environmental nuisances from industrial activities in residential areas of Arab municipalities in Israel", en Applied Geography, 35, p. 353-362.

- Tuan, Y. (1974). Topophilia: A study of environmental perception. Attitudes and values. Nueva York: Prentice-Hall.

- Valera, S., Pol, E. y Vidal, T. (s.f.). Elementos básicos de psicología ambiental. Universitat de Barcelona. Disponible en http://www.ub.edu/dppss/psicamb/

- Warring, R. (1983). Handbook of noise and vibration control. Morden, Trade and Technical Press, $5^{\mathrm{a}}$ ed. 